\title{
Why New Spinal Cord Plasticity Does Not Disrupt Old Motor Behaviors
}

\author{
Yi Chen, ${ }^{1}$ Lu Chen, ${ }^{1}$ Yu Wang, ${ }^{1}$ Xiang Yang Chen, ${ }^{1,2}$ and Jonathan R. Wolpaw ${ }^{1,2,3,4}$ \\ ${ }^{1}$ National Center for Adaptive Neurotechnologies, Wadsworth Center, New York State Department Health, Albany, New York 12201-0509, ${ }^{2}$ Department of \\ Biomedical Sciences, State University of New York, Albany, New York 12222, 32Department of Neurology, Stratton VA Medical Center, Albany, New York \\ 12208, and ${ }^{4}$ Department of Neurology, Neurological Institute, Columbia University, New York, New York 10032
}

When new motor learning changes the spinal cord, old behaviors are not impaired; their key features are preserved by additional compensatory plasticity. To explore the mechanisms responsible for this compensatory plasticity, we transected the spinal dorsal ascending tract before or after female rats acquired a new behavior-operantly conditioned increase or decrease in the right soleus H-reflex - and examined an old behavior-locomotion. Neither spinal dorsal ascending tract transection nor H-reflex conditioning alone impaired locomotion. Nevertheless, when spinal dorsal ascending tract transection and H-reflex conditioning were combined, the rats developed a limp and a tilted posture that correlated in direction and magnitude with the H-reflex change. When the right $\mathrm{H}-\mathrm{reflex}$ was increased by conditioning, the right step lasted longer than the left and the right hip was higher than the left; when the right $\mathrm{H}$-reflex was decreased by conditioning, the opposite occurred. These results indicate that ascending sensory input guides the compensatory plasticity that normally prevents the plasticity underlying H-reflex change from impairing locomotion. They support the concept of the state of the spinal cord as a negotiated equilibrium that reflects the concurrent influences of all the behaviors in an individual's repertoire; and they support the new therapeutic strategies this concept introduces.

Key words: H-reflex; motor learning; operant conditioning; plasticity; rehabilitation; spinal cord

\section{Significance Statement}

The spinal cord provides a reliable final common pathway for motor behaviors throughout life. Until recently, its reliability was explained by the assumption that it is hardwired; but it is now clear that the spinal cord changes continually as new behaviors are acquired. Nevertheless, old behaviors are preserved. This study shows that their preservation depends on sensory feedback from the spinal cord to the brain: if feedback is removed, the acquisition of a new behavior may disrupt an old behavior. In sum, when a new behavior changes the spinal cord, sensory feedback to the brain guides further change that preserves old behaviors. This finding contributes to a new understanding of spinal cord function and to development of new rehabilitation therapies.

\section{Introduction}

It has long been commonly assumed that learning depends entirely on plasticity in the brain. Nevertheless it is now clear that the neural substrate of a motor behavior often includes plasticity in the spinal cord as well (Wolpaw and Tennissen, 2001; Doyon et

\footnotetext{
Received March 21, 2017; revised July 7, 2017; accepted July 14, 2017.

Author contributions: Y.C., Y.W., X.Y.C., and J.R.W. designed research; Y.C., L.C., Y.W., X.Y.C., and J.R.W. performed research; Y.C., L.C., Y.W., X.Y.C., and J.R.W. analyzed data; Y.C., X.Y.C., and J.R.W. wrote the paper.

This work was supported by the National Institutes of Health (HD36020. X.Y.C.; NS061823, X.Y.C. and J.R.W.; NS22189, J.R.W.; 1P41EB018783, J.R.W.; HD32571, A.W. English), and a VA Merit Award (1 101 BX002550, J.R.W.). We thank Drs. Jonathan S. Carp, Dennis J. McFarland, Aiko K. Thompson, and Elizabeth Winter Wolpaw for comments on the manuscript.

The authors declare no competing financial interests.

Correspondence should be addressed to Xiang Yang Chen, Wadsworth Center, New York State Department Health, Empire State Plaza, Albany, NY 12201-0509. E-mail: xiangyang.chen@health.ny.gov.

DOI:10.1523/JNEUROSCI.0767-17.2017

Copyright $\odot 2017$ the authors $\quad 0270-6474 / 17 / 378198-09 \$ 15.00 / 0$
}

al., 2009; Wolpaw, 2010, 2012; Dayan and Cohen, 2011; Penhune and Steele, 2012; Pierrot-Deseilligny and Burke, 2012; Vahdat et al., 2015). Since the spinal cord, together with its analogous brainstem nuclei, is the final common pathway for motor behaviors, the spinal cord plasticity associated with the acquisition of a new motor behavior can affect existing (i.e., old) behaviors that use the same spinal neurons and synapses. Despite this, new behaviors do not normally disrupt old ones; the normal CNS acquires and maintains an extensive repertoire of motor behaviors throughout life. Recent studies indicate that the brain and spinal cord plasticity associated with a new behavior includes compensatory plasticity that preserves the key features of old behaviors (e.g., right/left symmetry in locomotion; Chen et al., 2011, 2014a). Compensatory spinal cord plasticity may also occur when trauma impairs motor function (Frigon and Rossignol, 2009).

We hypothesize that sensory feedback to the brain guides this compensatory plasticity. When the spinal cord plasticity under- 
A

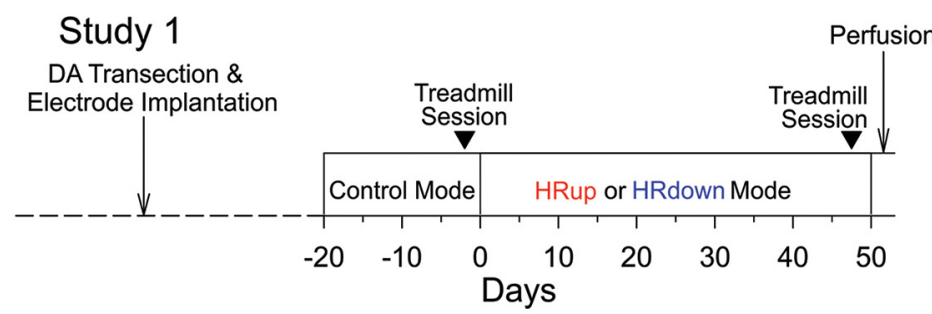

Study 2

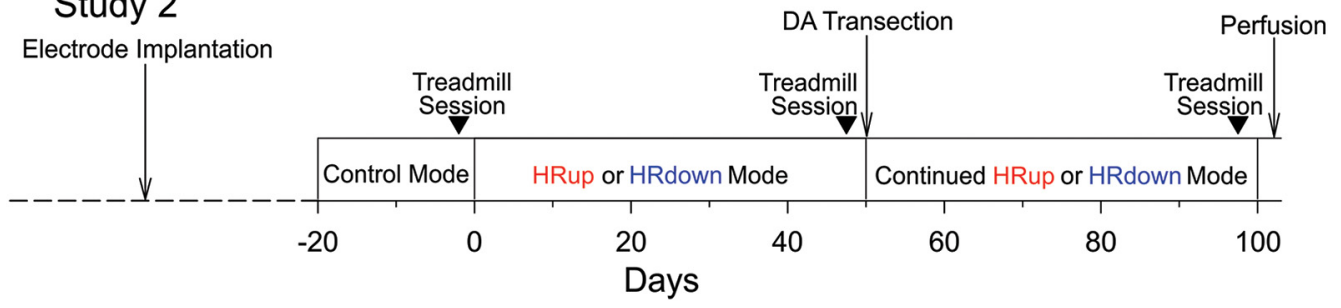

B
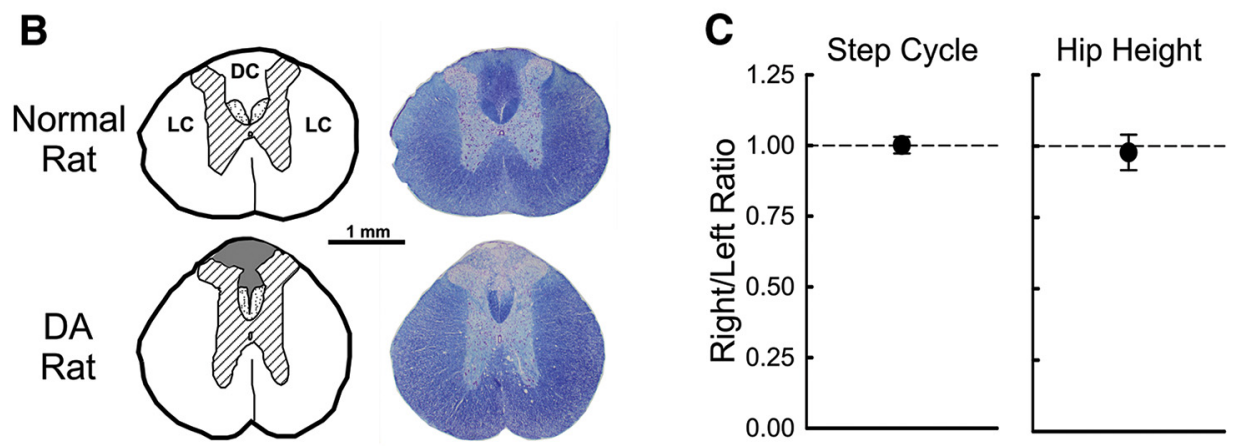

Figure 1. Study design. $\boldsymbol{A}$, Studies 1 and 2. The times of electrode implantation, dorsal ascending tract (DA) transection, H-reflex data collection, locomotor assessment on the treadmill, and perfusion are indicated. $\boldsymbol{B}$, Dorsal ascending tract transection. Camera lucida drawings and transverse sections of T9 spinal cord from a normal rat and a DA rat. DC, Dorsal columns; $L C$, lateral column; dotted areas at base of dorsal columns, (STs. The dorsal ascending tract transection is apparent; (STs and lateral columns are intact. C, Average ( \pm SD) values for right/left step symmetry and hip height symmetry for DA rats before H-reflex conditioning (1.0 is perfect symmetry). Dorsal ascending tract transection alone does not produce locomotor asymmetry.

lying a new behavior impairs an old behavior, the impairment is reflected in the sensory feedback associated with performance of the old behavior. Discrepancies between this feedback and the feedback associated with normal performance guide further plasticity, which reduces the discrepancies and preserves the key features of the old behavior. This hypothesis predicts that the loss of sensory feedback to the brain will allow the spinal cord plasticity underlying a new behavior to impair an old behavior.

We tested this prediction by examining the impact of a new motor behavior - an operantly conditioned increase or decrease in the soleus H-reflex (Wolpaw, 1987; Chen and Wolpaw, 1995; Thompson et al., 2009)—on an old motor behavior-locomotion - in rats in which midthoracic transection of the dorsal column ascending tract (DA rats) had greatly reduced proprioceptive feedback to the brain (Sengul and Watson, 2014). Specifically, we assessed in DA rats the impact of H-reflex conditioning on two key features of locomotion: right/left symmetry in step-cycle timing and in hip height. In normal rats, H-reflex conditioning does not disturb these symmetries; that is, it does not cause the rats to limp or to walk with a tilted posture (Chen et al., 2005, 2011). In DA rats, we found that the effects of H-reflex conditioning are substantially different.

\section{Materials and Methods}

Experimental design. Subjects were 32 young-adult female Sprague Dawley rats weighing $214-266 \mathrm{~g}$ (mean, $237 \mathrm{~g} ; \mathrm{SD}, \pm 15 \mathrm{~g}$ ) at the beginning of the study. The use of female rats reduced the need for bladder expression in the first few days after dorsal column ascending tract transection. H-reflex conditioning is comparable in females and males (Chen and Wolpaw, 1995, 1997, 2002; Chen et al., 1996, 2005, 2014a). All procedures satisfied the Guide for the Care and Use of Laboratory Animals (National Academies Press, Washington, D.C., 2011) and had been reviewed and approved by the Institutional Animal Care and Use Committee of the Wadsworth Center.

Figure $1 A$ shows the two studies. In Study 1, we transected the dorsal column ascending tract at thoracic level $\mathrm{T} 9$ and then exposed rats to up-conditioning or down-conditioning of the right soleus H-reflex for the standard $50 \mathrm{~d}$ period, or to no conditioning. In Study 2, we exposed rats to H-reflex up-conditioning or down-conditioning for $50 \mathrm{~d}$, transected the dorsal column ascending tract, and then continued the conditioning for 50 more days. We assessed the impact of these procedures on locomotor right/left symmetries during walking on a treadmill.

The procedures for electrode implantation, dorsal column ascending tract transection, postsurgical care, assessment of locomotion, H-reflex conditioning, histological evaluation, and data analysis have been described previously (for review, see Chen and Wolpaw, 2012; Thompson and Wolpaw, 2014). They are summarized here.

Electrode implantation. Rats were implanted under general anesthesia [ketamine $\mathrm{HCl}(80 \mathrm{mg} / \mathrm{kg}$, i.p.) and xylazine $(10 \mathrm{mg} / \mathrm{kg}$, i.p. $)$ ] and aseptic conditions with chronic stimulating and recording electrodes as described previously (Chen and Wolpaw, 1995, 1997, 2002; Chen et al., 2001, 2002, 2005, 2006). To elicit the right soleus H-reflex, a silicone rubber nerve cuff containing a pair of fine-wire electrodes was placed around the right posterior tibial (PT) nerve just above the triceps surae branches. The cuff was closed by a suture that encircled the cuff. To record electromyographic (EMG) activity, a pair of fine-wire EMG electrodes with final $0.5 \mathrm{~cm}$ segments stripped and separated by $0.2-0.3 \mathrm{~cm}$ 
were placed in the right soleus muscle. The wires from the nerve cuff and the muscle were led subcutaneously to a connector plug mounted on the skull. In addition, small $(2 \mathrm{~mm})$ dots were tattooed bilaterally on the skin over the lateral aspects of the knees, hips (i.e., trochanter major), and iliac crests at the fifth lumbar vertebra to guide placement of markers during locomotor sessions (see below).

After surgery, the rat was kept warm and given an analgesic (Demerol, $0.2 \mathrm{mg}$, i.m.), and was returned to its cage and allowed to eat and drink ad libitum (Chen and Wolpaw, 1995, 2002).

Spinal cord dorsal column ascending tract transection. Before H-reflex conditioning (Study 1), or after a $50 \mathrm{~d}$ exposure to H-reflex upconditioning or down-conditioning (Study 2), each rat received a spinal cord dorsal ascending tract transection at $\mathrm{T} 9$ as described previously (Chen et al., 2001; Chen and Wolpaw, 2002). Briefly, under the anesthesia regimen described above, a partial dorsal laminectomy was performed at T8-9 with minimal disturbance of the dural envelope. The animal was then mounted on a stereotaxic frame with the nearby dorsal processes rigidly fixed. The $\mathrm{T} 9$ transection was performed by electrocautery. The cauterizer, mounted on the stereotaxic frame, was activated in brief pulses to minimize thermal damage to adjacent tissue. The transection extended $0.4 \mathrm{~mm}$ to either side of the midline and $0.7 \mathrm{~mm}$ into the spinal cord from the dorsal surface; thus, it cut the dorsal ascending tract bilaterally and left the underlying corticospinal tract (CST) wholly or largely intact. After transection, the site was rinsed with normal saline and covered with Durafilm to minimize connective tissue adhesions to the dura. The muscle and skin were then sutured in layers. Care in the days immediately after the transection included analgesia, antibiotics, bladder expression, and high-calorie dietary supplementation as previously described in detail (Chen and Wolpaw, 1997, 2002; Chen et al., 2001, 2006, 2014a, 2014b). Bladder function returned 1-3 d (mean, $1.3 \mathrm{~d}$; $\mathrm{SD}, \pm 0.6 \mathrm{~d})$ after transection. Open-field locomotion was assessed according to the rating scale of Basso et al. [1995; i.e., the BBB (Basso, Beattie, and Bresnahan) test; perfect score 21]. In all animals, the BBB score returned to $\geq 20$ in $2-6 \mathrm{~d}$ (mean, $3.4 \mathrm{~d} ; \mathrm{SD}, \pm 1.0 \mathrm{~d}$ ). Thus, although kinematic analysis (see below) focused on the hindlimbs and did not assess the possible effects of dorsal ascending tract transection on forelimb/hindlimb coordination (English, 1980, 1989), it was clear that dorsal ascending tract transection did not produce a persistent visible disturbance of locomotion.

Soleus H-reflex monitoring and conditioning. Data collection started $\geq 30 \mathrm{~d}$ after electrode implantation and continued $24 \mathrm{~h} / \mathrm{d}, 7 \mathrm{~d} /$ week for $\geq 70 \mathrm{~d}$ (Study 1) or $120 \mathrm{~d}$ (Study 2; Fig. 1A). During this period, each rat lived in a standard rat cage with a $40 \mathrm{~cm}$ flexible cable attached to the skull plug. The cable, which allowed the animal to move freely about the cage, conveyed the wires from the electrodes to a commutator above the cage, and from there to an EMG amplifier and a stimulus isolation unit. The rat had ad libitum access to water and food, except that during H-reflex conditioning it received food mainly by performing the task described below. Animal well-being was carefully checked several times each day, and body weight was measured weekly. Laboratory lights were dimmed from 9:00 P.M. to 6:00 A.M. each day.

Stimulus delivery and data collection were under the control of a computer system, which monitored ongoing EMG activity (gain, 1000×; bandpass, 100-1000 Hz; sample rate, $5000 \mathrm{~Hz}$ ) continuously $24 \mathrm{~h} / \mathrm{d}$, $7 \mathrm{~d}$ /week, for the entire period of data collection. Whenever the absolute value (equivalent to the full-wave rectified value) of right soleus background (i.e., ongoing) EMG activity remained within a defined range for a randomly varying $2.3-2.7 \mathrm{~s}$ period, the computer initiated a trial. In each trial, the computer stored the most recent 50 ms of EMG activity from the muscle (i.e., the background EMG interval), delivered a monophasic stimulus pulse to the nerve cuff, and stored the EMG activity for another $100 \mathrm{~ms}$. Stimulus pulse amplitude and duration were initially set to produce a maximum $\mathrm{H}$-reflex and an $\mathrm{M}$ response (direct motor response) that was typically just above threshold. (The direct motor response is the EMG activity produced by the few motorneuron axons directly excited by the nerve cuff stimulus.) In each rat, pulse duration remained fixed (typically $0.5 \mathrm{~ms}$ ) throughout study. Pulse amplitude was adjusted by the computer after each trial to maintain the right soleus $\mathrm{M}$ response [i.e., average absolute value of EMG activity in the M-response interval (typically 1.5-4.0 ms after PT nerve stimulation)] unchanged at a target size. This ensured that the effective strength of the nerve stimulus was stable throughout the study despite any changes that occurred in nerve-cuff electrode impedances or in other factors (Wolpaw, 1987; Chen and Wolpaw, 1995). H-reflex size was calculated as average absolute value of EMG activity in the H-reflex interval (typically 5.5-10.0 ms after stimulation) minus average absolute value of background EMG activity at the time of stimulation, and was expressed in units of average absolute value of background EMG activity at the time of stimulation.

To determine the initial size of the H-reflex, data were collected for $20 \mathrm{~d}$ under the control mode, in which the computer simply digitized and stored soleus EMG activity for $100 \mathrm{~ms}$ following the stimulus. Then, the rat was exposed to right soleus $\mathrm{H}$-reflex up-conditioning (HRup rats) or down-conditioning (HRdown rats) for $50 \mathrm{~d}$ (Study 1) or $100 \mathrm{~d}$ (Study 2; Fig. $1 A$ ). Under the up-conditioning or down-conditioning mode, the computer gave a reward (i.e., a $20 \mathrm{mg}$ food pellet) $200 \mathrm{~ms}$ after PT nerve stimulation if the absolute value of right soleus EMG activity in the H-reflex interval was above (up-conditioning) or below (down-conditioning) a criterion value. The criterion value was set and adjusted as needed each day so that the rat received an amount of food that met its daily requirement [e.g., $\sim 700$ reward pellets (i.e., $14 \mathrm{~g}$ ) per day for a $300 \mathrm{~g}$ rat].

In every rat, the impact on $\mathrm{H}$-reflex size of the initial $50 \mathrm{~d}$ of conditioning was determined by expressing the average daily $\mathrm{H}$-reflex size for Days 41-50 of conditioning in percentage of the average daily H-reflex size for the final 10 control-mode days. For example, a value of $150 \%$ indicated that the H-reflex had increased by $50 \%$. In the Study- 2 rats, the impact on H-reflex size of the second $50 \mathrm{~d}$ of conditioning (i.e., after dorsal ascending tract transection) was determined by expressing the average daily $\mathrm{H}$-reflex size for Days $91-100$ of conditioning in percentage of the average daily H-reflex size for Days 41-50.

Locomotor data collection. At the beginning of study, before electrode implantation, each rat learned to walk quadrupedally on a motor-driven treadmill at $9-13 \mathrm{~m} / \mathrm{min}$ over $1-2$ training sessions of $20-30 \mathrm{~min}$ each (Chen et al., 2005, 2006, 2011, 2014a, 2014b). These training sessions were effective: when the rats were placed on the treadmill for actual locomotor data collection later on, they typically walked immediately. Subsequently, locomotor data were collected from each rat in two (Study 1) or three (Study 2) treadmill sessions as shown in Figure $1 \mathrm{~A}$.

Before each locomotor session, the hindlimbs were shaved and, guided by the tattooed dots (see above), $3 \mathrm{~mm}$ reflective adhesive markers were placed on the lateral aspects of the fifth metatarsophalangeal joint, the ankle joint (i.e., lateral malleolus), the knee joint, the hip joint (i.e., trochanter major), the iliac crest at the fifth lumbar vertebra, and the midpoint between the ankle and knee joints of each leg to enable later analysis of locomotor kinematics. In each rat, treadmill speed was the same for all sessions. During locomotion, soleus EMG activity was continuously recorded (bandpass, $100-1000 \mathrm{~Hz})$, digitized $(4000 \mathrm{~Hz})$, and stored. In addition, locomotor kinematics were recorded bilaterally with a 3 -D video data-collection and analysis system (100 frames/s; Vicon Motion Systems). Data were collected under two conditions. One was undisturbed locomotion. In the other, the soleus H-reflex was elicited just after the middle of the stance phase (i.e., the "locomotor H-reflex") as described by Chen et al. (2005, 2006, 2014a, 2014b). Approximately 5 min (i.e., $~ 500$ step cycles) of data were collected under each condition. At the end of each session, the femur and tibia lengths in each leg were measured externally.

Analysis of locomotor data. The EMG activity recorded during undisturbed locomotion was rectified and used to calculate soleus locomotor $\mathrm{H}$-reflex size and (after low-pass filtering by a $50 \mathrm{~ms}$ running average) soleus locomotor burst amplitude. As for the H-reflex elicited in the conditioning protocol (i.e., the "protocol H-reflex"), locomotor H-reflex size was calculated as average absolute value of EMG activity in the $\mathrm{H}$-reflex interval minus average absolute value of background EMG activity at the time of stimulation, and was expressed in units of average absolute value of background EMG activity (Chen et al., 2005). Soleus locomotor burst amplitude was calculated as average absolute value of EMG activity between burst onset and offset and was expressed in microvolts (Chen et al., 2005, 2006).

The concurrent 3-D locomotor kinematic data were analyzed with Vicon Motus software (Vicon Motion Systems) to assess two measures 
that reflect the right/left symmetry of the step cycle. The first measure was step symmetry, defined as the average time from right foot contact (RFC) to left foot contact (LFC) divided by the average time from LFC and RFC (i.e., a value of 1.0 indicates that the step cycle is perfectly symmetrical). The second measure was hip-height symmetry, defined as the average right hip height during right stance divided by the average left hip height during left stance (i.e., a value of 1.0 indicates that the hip heights are equal). We selected these measures for two reasons. First, they are clearly key features of locomotion: if step symmetry differs from 1.0, the rat is limping; and if hip height differs from 1.0, the rat is tilted as it walks [furthermore, such asymmetries might have long-term musculoskeletal consequences (e.g., osteoarthritis in the spine or limbs)]. Second, in the absence of appropriate compensatory plasticity, these key features seemed likely to be impaired by unilateral conditioning of the soleus H-reflex (Chen et al., 2011). For these reasons, they appeared to provide a good test of our hypothesis that the dorsal column ascending tracts guide the compensatory plasticity that prevents H-reflex conditioning from impairing locomotion: the hypothesis predicts that, in DA rats, $\mathrm{H}$-reflex conditioning should produce asymmetry in the step cycle and in hip height.

Perfusion, postmortem examination, and lesion verification. At the end of data collection, each rat was anesthetized, the right soleus muscle was injected with cholera toxin subunit B-conjugated Alexa Fluor 488 (for retrograde labeling of soleus motoneurons), and $3 \mathrm{~d}$ later the rat was perfused through the heart for postmortem examination and lesion verification, and for later anatomical and immunohistochemical studies (Wang et al., 2006, 2009).

The nerve cuff, EMG electrodes, and tibial nerve were examined, and the right and left soleus muscles were removed and weighed. The femur and tibia were exposed and their lengths were measured. The spinal cord was removed and blocks, including the transection, were embedded in OCT (optimum cutting temperature) compound (Tissue-Tek) and frozen on dry ice. Transverse $20-\mu \mathrm{m}$-thick serial sections from these blocks were cut with a cryostat, mounted onto precoated glass slides (Superfrost, Thermo Fisher Scientific), and analyzed to define the location and size of the dorsal ascending tract transection and any associated damage to the CST or other tracts (Chen and Wolpaw, 1997, 2002; Chen et al., 2002).

Statistical analysis. The data consist of protocol H-reflexes (i.e., H-reflex sizes measured throughout the day whenever the rats satisfied the background EMG criteria), locomotor H-reflexes (i.e., H-reflex sizes measured during the stance phase of locomotion), soleus locomotor EMG burst amplitudes, and putative key locomotor features (i.e., right/ left symmetry in step-cycle timing, right/left symmetry in hip height).

The first objective was to compare the impact of H-reflex up or down conditioning on these measures in normal rats to the impact in DA rats. We compared the rat groups (i.e., up-conditioned normal rats, downconditioned normal rats, unconditioned DA rats, up-conditioned DA rats, down-conditioned DA rats) by ANOVA. The normal rats were the Study-2 rats before dorsal ascending tract transection; the DA rats were the Study-1 rats (Fig. 1A). If the groups differed significantly, we then compared for each group the data before conditioning to those at the end of up-conditioning or down-conditioning or no conditioning by paired $t$ test.

The second objective was to evaluate for normal rats and for DA rats the correlations between conditioning-induced change in H-reflex size and changes in key locomotor features. The normal rats were the Study-2 rats before dorsal ascending tract transection; the DA rats were the Study-1 rats and the post-dorsal ascending tract transection Study-2 rats (Fig. 1A). These correlations were evaluated by linear regression.

\section{Results}

\section{Animal well-being and postmortem examination}

Animals remained healthy and active throughout data collection. Body weight increased from 214 to $266 \mathrm{~g}$ (mean, $237 \mathrm{~g}$; SD, $\pm 15 \mathrm{~g}$ ) at the beginning of the study to $295-459 \mathrm{~g}$ (mean, $347 \mathrm{~g}$; SD, $\pm 40 \mathrm{~g}$ ) at the time of perfusion. Right and left soleus muscle weights (measured as percentage of body weight) averaged 0.049\% (SD, $\pm 0.009 \%$ ) for the right and $0.048 \%$ (SD, $\pm 0.008 \%$ ) for the left. They did not differ significantly from each other $(p=0.39$ by paired $t$ test), and did not differ from soleus muscle weights of normal rats (Chen and Wolpaw, 1995, 1997, 2002; Chen et al., 1996, 1999, 2001, 2002, 2005, 2006). Examination of the nerve cuffs revealed the expected connective tissue investment of the wires and apparent good preservation of the nerve inside the cuff.

\section{Dorsal ascending tract transection}

Quantitative analysis indicated that dorsal ascending tract transection was usually complete or nearly complete in most of the rats. On average, $19 \%$ (SD, $\pm 20 \%$; range, $0-67 \%$ ) of the right and $16 \%$ (SD, $\pm 19 \%$; range, $0-60 \%$ ) of the left dorsal ascending tract remained. Figure $1 B$ shows a T9 transverse section from a representative rat. As Figure $1 C$ also shows, dorsal ascending tract transection itself did not disturb the normal right/left symmetries in step-cycle timing or hip height during locomotion.

The right and left lateral columns were entirely intact in all 32 rats; and the right and left CSTs were entirely intact in 26 rats. Six rats had sustained CST damage, indicating that the transection had extended too deep. This CST damage is potentially important because the CST is essential for acquisition and maintenance of H-reflex down-conditioning and for acquisition of up-conditioning (Chen and Wolpaw, 2002; Chen et al., 2002, 2003). In these six rats, the percentages of the right and left CST that remained were 100 and $69 \%, 88$ and $68 \%, 68$ and 100\%, 43 and $38 \%, 11$ and $31 \%$, and 0 and $0 \%$, respectively. The latter three rats, with large or complete CST lesions, were excluded from the data analysis.

\section{The rats studied}

The Study-1 results include data from 18 DA rats, 6 exposed to H-reflex up-conditioning, 6 to down-conditioning, and 6 to no conditioning (NC). The Study-2 results include data from 10 rats exposed to either up-conditioning ( 5 rats) or down-conditioning ( 5 rats) before dorsal ascending tract transection and then continued to be conditioned for another $50 \mathrm{~d}$ (one additional Study-2 down-conditioned rat did not finish the study due to breakage of the nerve-cuff wires).

For the purpose of this investigation (to determine whether the loss of sensory feedback to the brain caused by dorsal ascending tract transection allows the spinal cord plasticity underlying the new behavior of H-reflex conditioning to impair the old behavior of locomotion), the results provide three sets of data. The data gathered from the Study-2 rats before dorsal ascending tract transection assess the impact of $\mathrm{H}$-reflex conditioning on locomotion in normal rats. The data gathered from the Study-1 rats assess the impact of H-reflex conditioning on locomotion in rats in which the dorsal ascending tract was transected before conditioning. Finally, the data gathered from the Study-2 rats after dorsal ascending tract transection assess the impact of further H-reflex conditioning on locomotion in rats in which the dorsal ascending tract was transected after the initial $50 \mathrm{~d}$ of H-reflex conditioning had increased or decreased the H-reflex.

\section{H-reflex conditioning}

As Figure $2 A$ shows, conditioning had its expected effects on the right soleus H-reflex in both the DA rats of Study 1 and the notyet-transected (i.e., normal) rats of Study 2: H-reflex size changed gradually in the rewarded direction over the $50 \mathrm{~d}$ period of upconditioning (HRup rats) or down-conditioning (HRdown rats). Down-conditioning decreased the H-reflexes of the Study-2 rats somewhat more than those of the Study-1 rats, although this 


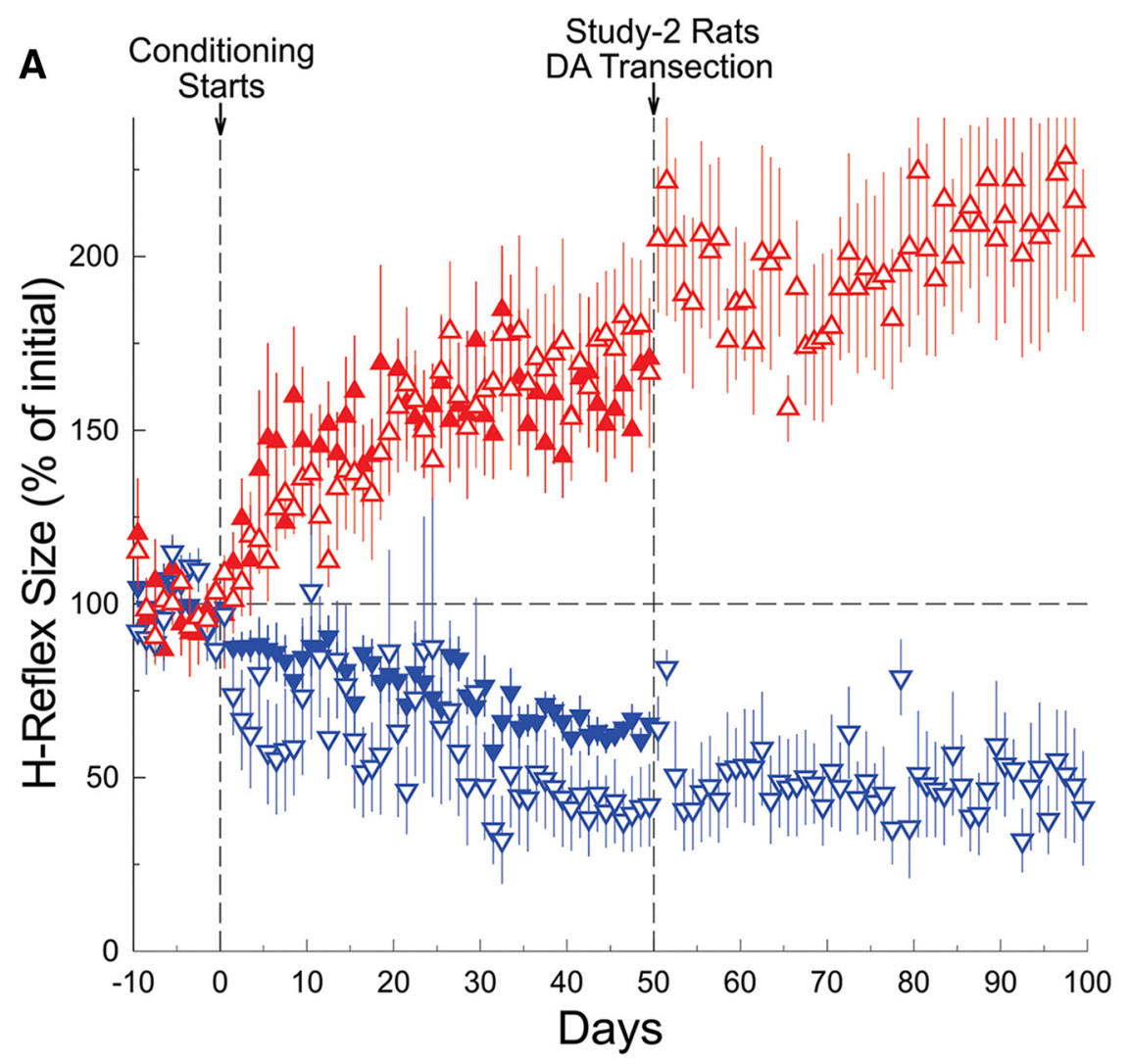

B
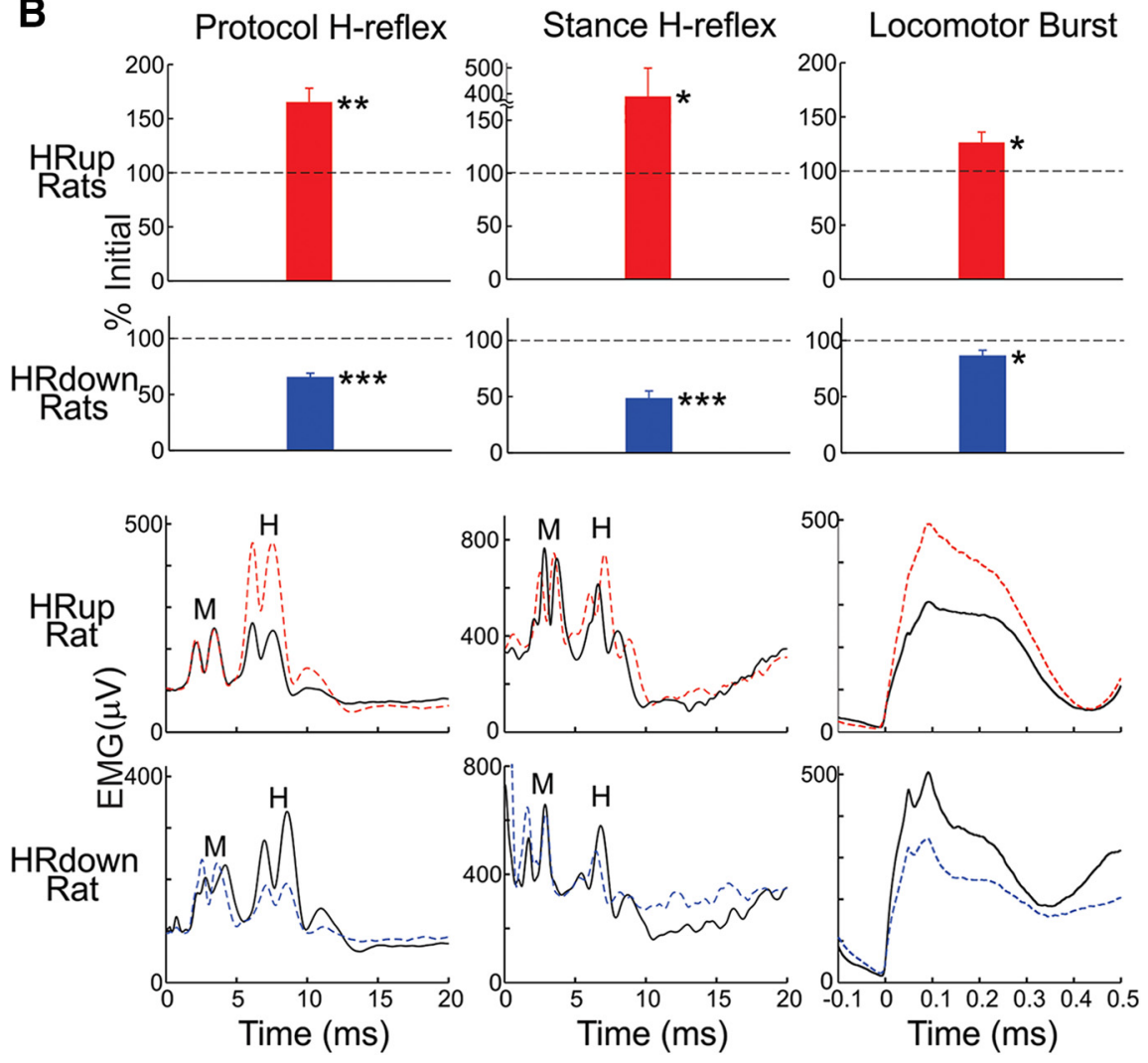

Figure 2. H-reflex conditioning and its effects on soleus locomotor EMG activity. $\boldsymbol{A}$, Average ( $\pm \mathrm{SE}$ ) H-reflex size (in percentage of average control value) for HRup rats (red up-triangles) and HRdown rats (blue down-triangles) of Study 1 (solid symbols) and Study 2 (open symbols) for each day during the final $10 \mathrm{~d}$ of the control-mode period and the 50 (Study 1) or $100 \mathrm{~d}$ (Study 2) of HRup or HRdown conditioning. Dorsal ascending tract transection in the Study-2 rats is indicated. $\boldsymbol{B}$, Effects of H-reflex conditioning on $\mathrm{H}$-reflexes and locomotor bursts in DA rats. Top: Average ( $\pm \mathrm{SE}$ ) protocol H-reflexes (i.e., the H-reflexes measured in the difference did not reach significance ( $p=$ 0.06 by $t$ test). In neither the Study- 1 rats nor the Study-2 rats did the average $\mathrm{H}$-reflex increases (or decreases) in the final $10 \mathrm{~d}$ of the $50 \mathrm{~d}$ period of upconditioning (or down-conditioning) differ significantly from those in the many normal rats studied previously (for review, see Chen and Wolpaw, 2012; Thompson and Wolpaw, 2014).

After the Study-2 rats underwent dorsal ascending tract transection and conditioning continued for another $50 \mathrm{~d}$, the H-reflex tended to rise further in the HRup rats ( $p=0.07$ by paired $t$ test) and remained approximately the same in the HRdown rats (in the Study-2 rats, the transient increase 1-2 d after dorsal ascending tract transection is a nonspecific effect of the surgery and/or anesthesia; Chen et al., 2001).

\section{Locomotor H-reflexes and soleus locomotor EMG activity}

In the DA rats of Study 1 and the not-yettransected (i.e., normal) rats of Study 2, the change in the H-reflex elicited during the conditioning protocol (i.e., the protocol H-reflex) was accompanied by corresponding change in the locomotor H-reflex (i.e., the H-reflex elicited during the stance phase of locomotion) and in the right soleus locomotor burst, which supports stance. Figure $2 B$ summarizes and illustrates these effects. Thus, in DA rats as in normal rats, $\mathrm{H}$-reflex conditioning changed the soleus contribution to locomotion.

\section{Locomotor symmetry}

Despite their similarity in the effects of $\mathrm{H}$-reflex conditioning on H-reflexes and soleus locomotor EMG activity, normal rats and DA rats differed markedly in the impact of conditioning on locomotor symmetry. Figure $3 A$ summarizes these results. As expected in normal rats (Chen et al., 2005, 2011), H-reflex conditioning did not affect the normal right/left symmetries in step-cycle timing or hip height

\section{$\leftarrow$}

conditioning protocol), locomotor H-reflexes (i.e., the $\mathrm{H}$-reflexes measured in the stance phase of locomotion), and soleus locomotor bursts for the DA rats of Study 1 at the end of HRup or HRdown conditioning ${ }^{* * *} p<0.001,{ }^{* *} p<0.01$, ${ }^{*} p<0.05$ vs initial (i.e., preconditioning) value by paired $t$ test). Bottom, Data from representative HRup and HRdown DA rats. Average post-stimulus EMG activity in the conditioning protocol (left) or during locomotion (middle), and average locomotor bursts (right), in the control mode (solid) and after conditioning (dashed) are shown. M responses and $\mathrm{H}$-reflexes are indicated. The effects of conditioning on H-reflexes and locomotor bursts are evident. 

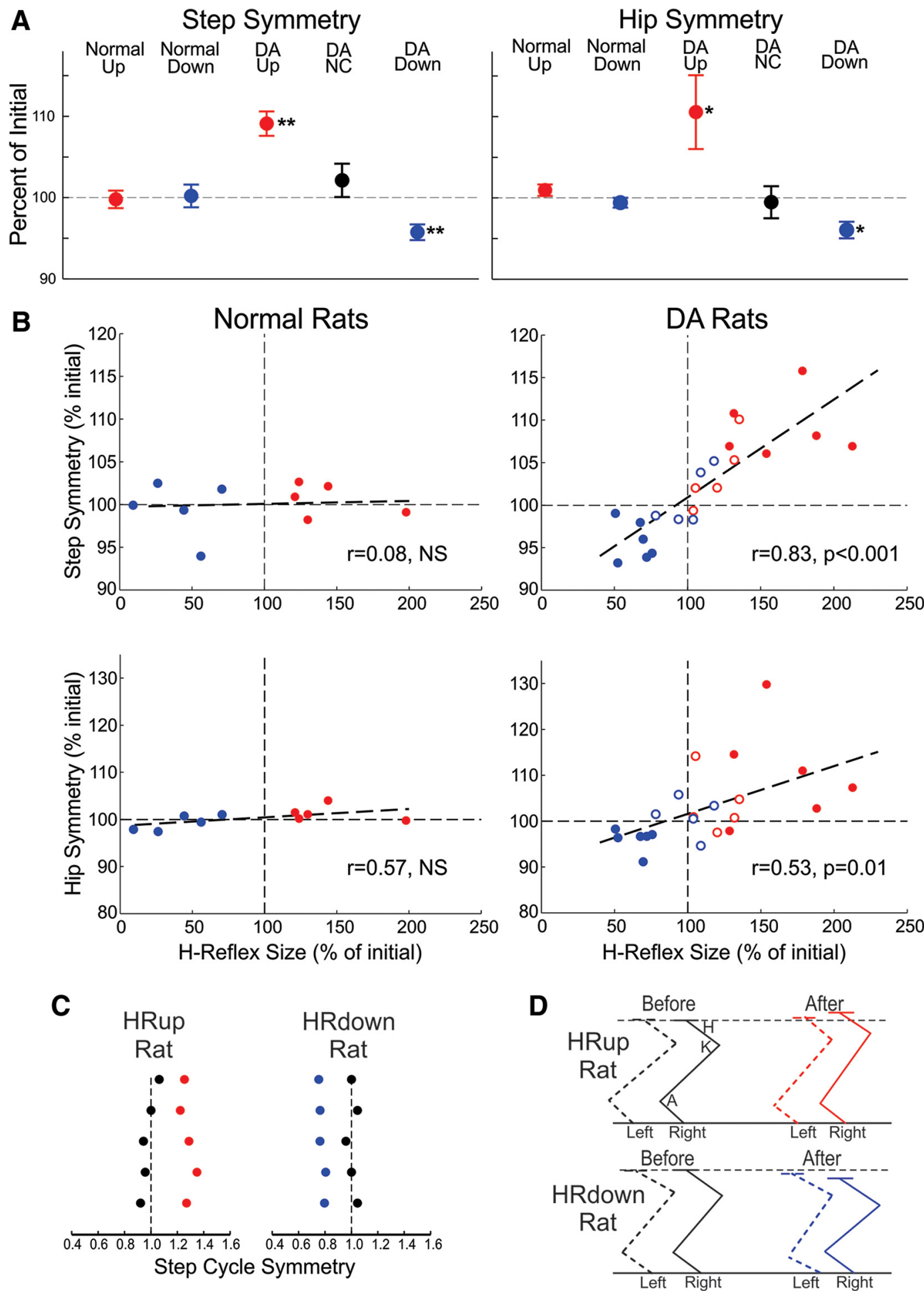

Figure 3. Impact of H-reflex conditioning on key locomotor features in normal rats and in DA rats. $A$, left, Average ( \pm SE) right/left step symmetry after up-conditioning or down-conditioning or no conditioning (NC; in percentage of initial value). In normal rats (Study-2 rats before dorsal ascending tract transection), conditioning does not affect symmetry. In DA rats (Study-1 rats), conditioning produces step asymmetry: up-conditioning increases and down-conditioning decreases the ratio of the time between RFC and LFC to the time between LFC and RFC. Right, Average $( \pm$ SE) right/left hip height symmetry at the end of up-conditioning or down-conditioning or no conditioning (in percentage of initial value). In normal rats, conditioning does not affect hip-height symmetry. In DA rats, the right hip is higher after up-conditioning and lower after down-conditioning ( ${ }^{* *} p<0.01,{ }^{*} p<0.05$ vs initial value by paired $t$ test). $B$, Step symmetry (top) and hip-height symmetry (bottom) versus final H-reflex size after up-conditioning (red) or down-conditioning (blue) for normal rats (left) and DA rats (right). The normal rats are the Study-2 rats before dorsal ascending tract transection. Their final $\mathrm{H}$-reflex size is their $\mathrm{H}$-reflex size at the end of the initial $50 \mathrm{~d}$ of conditioning just before dorsal ascending tract transection; it is expressed in percentage of their $\mathrm{H}$-reflex size for the control mode period before conditioning. The DA rats with solid symbols are the Study-1 rats. Their final H-reflex size is their H-reflex size at the end of the $50 \mathrm{~d}$ of conditioning; it is expressed in percentage of their $\mathrm{H}$-reflex size for the control-mode period before conditioning. The DA rats with open symbols are the Study-2 rats after dorsal ascending tract transection. Their final $\mathrm{H}$-reflex size is their $\mathrm{H}$-reflex size at the end of the second $50 \mathrm{~d}$ of conditioning; it is expressed in percentage of their $\mathrm{H}$-reflex size at the end of the initial $50 \mathrm{~d}$ of conditioning just before dorsal ascending tract transection. In normal rats, H-reflex up-conditioning or down-conditioning does not affect step or hip symmetry. In contrast, in DA rats transected before conditioning (solid symbols), conditioning creates asymmetries that correlate with the direction and magnitude of the $50 \mathrm{~d}$ of conditioning. Similarly, in DA rats transected after (Figure legend continues.) 
in the not-yet-transected Study-2 rats. After $50 \mathrm{~d}$ of up-conditioning or down-conditioning (i.e., just before dorsal ascending tract transection), right/left step-cycle timing and hip heights remained symmetrical. In contrast, in the DA rats of Study $1, \mathrm{H}$-reflex conditioning produced significant right/left asymmetries in step-cycle timing and hip heights that correlated with the direction of $\mathrm{H}$-reflex change. Upconditioning made the right step last longer than the left and it made the right hip higher than the left; down-conditioning made the right step briefer than the left and the right hip lower than the left. Furthermore, when, after $50 \mathrm{~d}$ of conditioning, the normal rats of Study 2 underwent dorsal ascending tract transection and continued to be conditioned for another $50 \mathrm{~d}$, those that developed further change in H-reflex size also developed corresponding asymmetries in step-cycle timing and hip height.

Figure $3 B$ shows for normal rats and DA rats the correlations between H-reflex change and right/left symmetries in step-cycle timing and hip height. In normal rats, H-reflex change had no detectable effect. In DA rats, H-reflex conditioning produced asymmetries in both measures that correlated with the direction and magnitude of the concurrent change in H-reflex size. Thus, the more the H-reflex increased with up-conditioning, the more the right step became longer than the left and the right hip became higher; and the more the H-reflex decreased with downconditioning, the more the right step became shorter than the left and the right hip became lower. Figure $3 C, D$ illustrates these effects.

In summary, H-reflex conditioning does not disturb locomotion in normal rats. In DA rats, it does disturb locomotion: the step-cycle asymmetry is manifested as a limp and the hip-height asymmetry is manifested as a tilted hindlimb posture. The direction and magnitude of these abnormalities correlate with the direction and magnitude of $\mathrm{H}$-reflex change.

\section{Discussion}

The primary significance of these results is their contribution toward a new understanding of spinal cord function. The work of recent decades has made it clear that the spinal cord changes throughout life as growth and aging occur, as new behaviors are acquired, and in response to trauma and disease (for review, see Wolpaw and Tennissen, 2001; Wolpaw, 2010, 2012; PierrotDeseilligny and Burke, 2012). Thus, the traditional concept of a hardwired spinal cord that does not change after early development is no longer viable. However, it has not been clear how a continually changing spinal cord remains a reliable final common pathway for all the behaviors in an individual's repertoire. The present results help answer this question. They thereby support a new concept of spinal cord function that reconciles its long-recognized reliability with its newly appreciated plasticity.

\footnotetext{
$\leftarrow$

(Figure legend continued.) $50 \mathrm{~d}$ of conditioning, the subsequent $50 \mathrm{~d}$ of conditioning creates asymmetries that correlate with any further change in $\mathrm{H}$-reflex size. C, Step-cycle symmetry in two DA rats of Study 1 before (black) and after up-conditioning (HRup rat; red) or downconditioning (HRdown rat; blue). Symmetry values for five consecutive step cycles are shown. Perfect symmetry is 1.0. Before conditioning, the step cycle is symmetrical in both rats; after conditioning, the right step is longer than the left in the HRup rat and shorter in the HRdown rat. Thus, the rats are limping. D, Average right and left hindlimb positions during stance in two DA rats before and after up-conditioning or down-conditioning. The rats walk toward the right. Hip $(H)$, knee $(K)$, and ankle $(A)$ angles are marked. Before conditioning, right and left hip heights are equal. After up-conditioning, the right hip is higher than the left; after down-conditioning, it is lower. Thus, the HRup rat tilts to the left; and the HRdown rat tilts to the right.
}

\section{Maintenance of motor behaviors}

The acquisition of a new motor behavior can certainly affect an old behavior. The distinctive walk of professional ballet dancers is a striking example (Kilgannon, 1996). The changes in spinal reflex pathways (Nielsen et al., 1993; and presumably in other pathways) that underlie the acquisition of this specialized behavior change the old behavior of locomotion. Nevertheless, locomotion is not disrupted; it looks different (i.e., its kinematics change), but it remains satisfactory. In rats, soleus H-reflex conditioning has an analogous impact. Because it changes the spinal pathway of the reflex (for review, see Wolpaw and Lee, 1989; Thompson and Wolpaw, 2014), it affects the pathway's contribution to other behaviors, such as locomotion. Locomotor EMG activity and kinematics change (Chen et al., 2005, 2011; Fig. 2B). Nevertheless, in normal rats, the features that characterize normal locomotion (e.g., right/left symmetry in step timing and hip height) are preserved. As Bernstein (1967) emphasized, motor behaviors have key features that are much more precisely controlled than other features. This principle has been formalized in the "uncontrolled manifold" concept, which partitions the variance in kinematic variables into the part that impairs key features (e.g., right/left step symmetry) and the part that does not (Scholz and Schöner, 1999; Latash et al., 2007; Chang et al., 2009).

Recent studies indicate that, when acquisition of a new behavior (i.e., soleus H-reflex conditioning) changes the lumbosacral spinal cord, the preservation of normal locomotion entails compensatory plasticity involving other spinal pathways (Chen et al., 2011, 2014a). The resulting changes in locomotor EMG activity and kinematics preserve key locomotor features (e.g., right/left symmetry in hip height). For example, when soleus H-reflex down-conditioning decreases ankle angle (i.e., decreases plantarflexion) during locomotion (which by itself would lower the hip), an associated increase in hip extension may preserve hip height (Chen et al., 2011).

Studies that take advantage of the anatomical separation between the brain and spinal cord illuminate the compensatory plasticity that preserves old behaviors. After denervation of an important leg muscle in cats, locomotion gradually recovers. By assessing the locomotor impact of subsequent spinal cord transection, Carrier et al. (1997) showed that this recovery depends on plasticity in both the brain and spinal cord. Frigon and Rossignol (2009) further extended this work. In another example, unilateral operant down-conditioning of the triceps surae H-reflex in monkeys has little or no effect on the contralateral H-reflex (Wolpaw et al., 1989, 1993). Terminal studies that assessed the impact of spinal cord transection showed that the preservation of an unchanged contralateral H-reflex depended on plasticity in both the brain and spinal cord (Wolpaw and Lee, 1989). Thus, just as the initial acquisition of a motor behavior may involve plasticity in both the brain and spinal cord, its subsequent preservation as further behaviors are acquired may do so as well. Each behavior rests on a network of brain and spinal plasticity that changes as needed to maintain the key features of the behavior.

The present results illuminate the process that maintains a motor behavior; they show that this process is driven and guided by sensory feedback from the spinal cord to the brain. Dorsal ascending tract transection, which eliminates much of that feedback, allows H-reflex conditioning to disrupt key locomotor features, such as step-cycle and hip-height symmetry, producing a limp and a tilted posture. After dorsal ascending tract transection, the brain no longer receives feedback reflecting the impact of H-reflex conditioning on locomotion; and/or the limited feed- 
back that it does receive is inadequate for guiding appropriate compensatory plasticity. The result is that appropriate compensation does not occur and locomotion becomes abnormal.

In a similar fashion, loss or distortion of ascending sensory feedback may contribute to the disabilities of people with spinal cord injury, stroke, or other disorders that impair interactions between the brain and the spinal cord. These disorders do not merely impair the brain's descending control over the spinal cord, they can also impair the ascending sensory feedback that guides this control. Thus, motor disabilities may occur and persist not only because the disorder creates them, but also because the disorder disrupts the feedback needed to guide their correction and/or because the limited feedback that remains is inadequate for guiding appropriate compensatory plasticity.

\section{Function of the spinal cord}

By demonstrating that dorsal ascending tract transection allows $\mathrm{H}$-reflex conditioning to disrupt locomotion, the present results help explain how the highly plastic spinal cord remains a reliable final common pathway for motor behaviors throughout life. We have recently proposed that new motor behaviors are acquired and old behaviors are preserved through a process in which the substrate of brain and spinal plasticity underlying each behavior operates as an independent agent: each behavior repeatedly induces spinal cord (and brain) plasticity that maintains its key features despite the plasticity induced by other behaviors (Wolpaw, 2010; Thompson et al., 2013; Chen et al., 2014a). Whenever the behavior is performed, the associated sensory feedback is compared with the expected feedback (i.e., the feedback associated with normal performance). This comparison (which may occur in the cerebellum; D'Angelo et al., 2016; Popa et al., 2016) detects deviations from the key features of the behavior. These deviations constitute error signals that guide appropriate changes in the brain and spinal substrate responsible for the behavior.

As a result, the spinal cord is continually modified by the many behaviors that use it. The aggregate process is a negotiation among the behaviors: they negotiate the properties of the spinal neurons and synapses that they all use. The negotiation ensures that spinal neuronal and synaptic properties are maintained in an equilibrium - a negotiated equilibrium - that serves all the behaviors in an individual's repertoire (Wolpaw, 2010; Thompson et al., 2013; Chen et al., 2014a). This model assumes that sensory feedback to the brain guides the plasticity that maintains each behavior despite the plasticity produced by other behaviors. Thus, the model predicts that loss of sensory feedback will allow the spinal cord plasticity underlying a new behavior to impair old behaviors. The present results confirm this prediction.

\section{Experimental and clinical uses of $\mathrm{H}$-reflex conditioning}

This demonstration of the importance of ascending sensory input in preserving an old behavior when new learning changes the spinal cord was made possible by a distinctive attribute of the H-reflex conditioning protocol. Unlike most motor learning, $\mathrm{H}$-reflex conditioning is not guided by the ascending somatosensory feedback generated during performance; it is guided principally by the food reward (i.e., by the visual, auditory, and/or gustatory input associated with the reward). Thus, H-reflex conditioning can occur in DA rats, in which its impact on old behaviors is apparent.

This attribute of the conditioning protocol underlies its promising therapeutic applications. In the present study, the protocol was used to disrupt normal locomotion. Applied in a clinical context, it represents a new therapeutic approach called “targeted neuroplasticity" (Thompson and Wolpaw, 2014, 2015). In rats in which a lateralized spinal cord injury has weakened stance on one side and created a limp, up-conditioning of the soleus $\mathrm{H}$-reflex on that side strengthens stance and restores locomotor symmetry (Chen et al., 2006). Similarly, in people with spasticity due to incomplete spinal cord injury, down-conditioning of the hyperactive soleus $\mathrm{H}$-reflex reduces limping and increases walking speed (Thompson et al., 2013). Furthermore, the global improvement in their walking cannot be attributed simply to the plasticity that reduces the H-reflex (i.e., the plasticity targeted by the reward contingency). The introduction of a new motor behavior-a smaller soleus $\mathrm{H}$-reflex-appears to trigger a wider process (i.e., a new negotiation) that leads to beneficial plasticity at many sites and improves locomotor muscle activity in the proximal and distal muscles of both legs (Thompson et al., 2013). Recent animal studies provide additional evidence for and insight into this initiation of wider beneficial plasticity (Chen et al., 2014b).

\section{Summary}

This study helps to explain how the highly plastic spinal cord reliably serves all the motor behaviors in an individual's repertoire. By showing that dorsal ascending tract transection allows the acquisition of a new behavior to disrupt an old behavior, the results support the conclusion that sensory feedback to the brain enables each behavior to exert continuing influence over spinal neuronal and synaptic properties and to thereby ensure its continued satisfactory performance.

\section{References}

Basso DM, Beattie MS, Bresnahan JC (1995) A sensitive and reliable locomotor rating scale for open field testing in rats. J Neurotrauma 12:1-21. CrossRef Medline

Bernstein NA (1967) The co-ordination and regulation of movements. Oxford: Pergamon.

Carrier L, Brustein E, Rossignol S (1997) Locomotion of the hindlimbs after neurectomy of ankle flexors in intact and spinal cats: model for the study of locomotor plasticity. J Neurophysiol 77:1979-1993. Medline

Chang YH, Auyang AG, Scholz JP, Nichols TR (2009) Whole limb kinematics are preferentially conserved over individual joint kinematics after peripheral nerve injury. J Exp Biol 212:3511-3521. CrossRef Medline

Chen XY, Wolpaw JR (1995) Operant conditioning of H-reflex in freely moving rats. J Neurophysiol 73:411-415. Medline

Chen XY, Wolpaw JR (1997) Dorsal column but not lateral column transection prevents down-conditioning of $\mathrm{H}$ reflex in rats. J Neurophysiol 78: 1730-1734. Medline

Chen XY, Wolpaw JR (2002) Probable corticospinal tract control of spinal cord plasticity in the rat. J Neurophysiol 87:645-652. CrossRef Medline

Chen XY, Wolpaw JR (2012) Operant conditioning of spinal cord reflexes in rats. In: Animal models of acute neurological injuries. II: injury and mechanistic assessments. (Chen J, Xu ZC, Xu XM, Zheng JH, eds), Chapter 44, 543-551. New York: Humana Press.

Chen XY, Wolpaw JR, Jakeman LB, Stokes BT (1996) Operant conditioning of H-reflex in spinal-cord injured rats. J Neurotrauma 13:755-766. CrossRef Medline

Chen XY, Wolpaw JR, Jakeman LB, Stokes BT (1999) Operant conditioning of H-reflex increase in spinal-cord injured rats. J Neurotrauma 16:175186. CrossRef Medline

Chen XY, Feng-Chen KC, Chen L, Stark DM, Wolpaw JR (2001) Short-term and medium-term effects of spinal cord tract transections on soleus H-reflex in freely moving rats. J Neurotrauma 18:313-327. CrossRef Medline

Chen XY, Carp JS, Chen L, Wolpaw JR (2002) Corticospinal tract transection prevents operantly conditioned increase of H-reflex in rats. Exp Brain Res 144:88-94. CrossRef Medline

Chen XY, Chen L, Wolpaw JR (2003) Conditioned H-reflex increase persists after transection of the main corticospinal tract in rats. J Neurophysiol 90:3572-3578. CrossRef Medline 
Chen Y, Chen XY, Jakeman LB, Schalk G, Stokes BT, Wolpaw JR (2005) The interaction of a new motor skill and an old one: $\mathrm{H}$-reflex conditioning and locomotion in rats. J Neurosci 25:6898-6906. CrossRef Medline

Chen Y, Chen XY, Jakeman LB, Chen L, Stokes BT, Wolpaw JR (2006) Operant conditioning of $\mathrm{H}$-reflex can correct a locomotor abnormality after spinal cord injury in rats. J Neurosci 26:12537-12543. CrossRef Medline

Chen Y, Chen L, Wang Y, Wolpaw JR, Chen XY (2011) Operant conditioning of rat soleus H-reflex oppositely affects another H-reflex and changes locomotor kinematics. J Neurosci 31:11370-11375. CrossRef Medline

Chen Y, Chen L, Liu R, Wang Y, Chen XY, Wolpaw JR (2014a) Locomotor impact of beneficial or non-beneficial $\mathrm{H}$-reflex conditioning after spinal cord injury. J Neurophysiol 111:1249-1258. CrossRef Medline

Chen Y, Chen L, Wang Y, Wolpaw JR, Chen XY (2014b) Persistent beneficial impact of H-reflex conditioning in spinal cord injured rats. J Neurophysiol 112:2374-2381. CrossRef Medline

D’Angelo E, Mapelli L, Casellato C, Garrido JA, Luque N, Monaco J, Prestori F, Pedrocchi A, Ros E (2016) Distributed circuit plasticity: new clues for the cerebellar mechanisms of learning. Cerebellum 15:139-151. DOI 10.1007/s12311-015-0711-7. CrossRef Medline

Dayan E, Cohen LG (2011) Neuroplasticity subserving motor skill learning. Neuron 72:443-454. CrossRef Medline

Doyon J, Bellec P, Amsel R, Penhune V, Monchi O, Carrier J, Lehéricy S, Benali H (2009) Contributions of the basal ganglia and functionally related brain structures to motor learning. Behav Brain Res 199:61-75. CrossRef Medline

English AW (1980) Interlimb coordination during stepping in the cat: effects of dorsal column section. J Neurophysiol 44:270-279. Medline

English AW (1989) Interlimb coordination during locomotion. Integ Compar Biol 29:255-266.

Frigon A, Rossignol S (2009) Partial denervation of ankle extensors prior to spinalization in cats impacts the expression of locomotion and the phasic modulation of reflexes. Neuroscience 158:1675-1690. CrossRef Medline

Kilgannon C (1996) By their walk shall you know them. New York Times, November 10. Available at: http://www.nytimes.com/1996/11/10/ nyregion/by-their-walk-shall-you-know-them.html

Latash ML, Scholz JP, Schöner G (2007) Toward a new theory of motor synergies. Motor Control 11:276-308. CrossRef Medline

Nielsen J, Crone C, Hultborn H (1993) H-reflexes are smaller in dancers from the Royal Danish Ballet than in well-trained athletes. Eur J Appl Physiol Occup Physiol 66:116-121. CrossRef Medline

Penhune V, Steele CJ (2012) Parallel contributions of cerebellar, striatal and M1 mechanisms to motor sequence learning. Behav Brain Res 226:579591. CrossRef Medline

Pierrot-Deseilligny E, Burke D (2012) The circuitry of the human spinal cord: spinal and corticospinal mechanisms of movement. Cambridge, UK: Cambridge UP.
Popa LS, Streng ML, Hewitt AL, Ebner TJ (2016) The errors of our ways: understanding error representations in cerebellar-dependent motor learning. Cerebellum 15:93-103. CrossRef Medline

Scholz JP, Schöner G (1999) The uncontrolled manifold concept: identifying control variables for a functional task. Exp Brain Res 126:289-306. CrossRef Medline

Sengul G, Watson C (2014) Ascending and descending pathways in the spinal cord. In: The rat nervous system, Chap 8 (Paxinos G, ed), pp 115-130. Amsterdam: Elsevier.

Thompson AK, Wolpaw JR (2014) Operant conditioning of spinal reflexes: from basic science to clinical therapy. Front Integr Neurosci 8:25. CrossRef Medline

Thompson AK, Wolpaw JR (2015) Targeted neuroplasticity for rehabilitation. Prog Brain Res 218:157-172. CrossRef Medline

Thompson AK, Chen XY, Wolpaw JR (2009) Acquisition of a simple skill: task-dependent adaptation plus long-term change in the human soleus H-reflex. J Neurosci 29:5784-5792. CrossRef Medline

Thompson AK, Pomerantz FR, Wolpaw JR (2013) Operant conditioning of a spinal reflex can improve locomotion after spinal cord injury in humans. J Neurosci 33:2365-2375. CrossRef Medline

Vahdat S, Lungu O, Cohen-Adad J, Marchand-Pauvert V, Benali H, Doyon J (2015) Simultaneous brain-cervical cord fMRI reveals intrinsic spinal cord plasticity during motor sequence learning. PLoS Biol 13: e1002186. CrossRef Medline

Wang Y, Pillai S, Wolpaw JR, Chen XY (2006) Motor learning changes GABAergic terminals on spinal motoneurons in normal rats. Eur J Neurosci 23:141-150. CrossRef Medline

Wang Y, Pillai S, Wolpaw JR, Chen XY (2009) H-reflex down-conditioning greatly increases the number of identifiable GABAergic interneurons in rat ventral horn. Neurosci Lett 452:124-129. CrossRef Medline

Wolpaw JR (1987) Operant conditioning of primate spinal reflexes: the H-reflex. J Neurophysiol 57:443-459. Medline

Wolpaw JR (2010) What can the spinal cord teach us about learning and memory? Neuroscientist 16:532-549. CrossRef Medline

Wolpaw JR (2012) Harnessing neuroplasticity for clinical applications. Brain 135:e215. CrossRef Medline

Wolpaw JR, Lee CL (1989) Memory traces in primate spinal cord produced by operant conditioning of H-reflex. J Neurophysiol 61:563-572. Medline

Wolpaw JR, Tennissen AM (2001) Activity-dependent spinal cord plasticity in health and disease. Annu Rev Neurosci 24:807-843. Medline

Wolpaw JR, Lee CL, Calaitges JG (1989) Operant conditioning of primate triceps surae H-reflex produces reflex asymmetry. Exp Brain Res 75:3539. Medline

Wolpaw JR, Herchenroder PA, Carp JS (1993) Operant conditioning of the primate H-reflex: factors affecting the magnitude of change. Exp Brain Res 97:31-39. CrossRef Medline 